\title{
Sputter-Deposited Ag Nanoparticles on Electrospun PCL Scaffolds: Morphology, Wettability and Antibacterial Activity
}

\author{
Daniele Valerini $^{1, *(\mathbb{D})}$, Loredana Tammaro ${ }^{2, *}$ (D) , Roberta Vitali ${ }^{3, *}$, Gloria Guillot ${ }^{4}$ and Antonio Rinaldi ${ }^{5, *(D)}$ \\ 1 SSPT-PROMAS-MATAS, ENEA—Italian National Agency for New Technologies, Energy and Sustainable \\ Economic Development, S.S. 7 Appia, km 706, 72100 Brindisi, Italy \\ 2 SSPT-PROMAS-NANO, ENEA-Italian National Agency for New Technologies, Energy and Sustainable \\ Economic Development, Piazzale E. Fermi, 1, Portici, 80055 Napoli, Italy \\ 3 SSPT-TECS-TEB, ENEA-Italian National Agency for New Technologies, Energy and Sustainable Economic \\ Development, Via Anguillarese 301, 00123 Rome, Italy \\ 4 NANOFABER srl, Via Anguillarese 301, 00123 Rome, Italy; gloria.guillot@nanofaber.com \\ 5 SSPT-PROMAS-MATPRO, ENEA-Italian National Agency for New Technologies, Energy and Sustainable \\ Economic Development, Via Anguillarese 301, 00123 Rome, Italy \\ * Correspondence: daniele.valerini@enea.it (D.V.); loredana.tammaro@enea.it (L.T.); \\ roberta.vitali@enea.it (R.V.); antonio.rinaldi@enea.it (A.R.)
}

Citation: Valerini, D.; Tammaro, L.; Vitali, R.; Guillot, G.; Rinaldi, A. Sputter-Deposited Ag Nanoparticles on Electrospun PCL Scaffolds: Morphology, Wettability and Antibacterial Activity. Coatings 2021, 11, 345. https://doi.org/10.3390/ coatings11030345

Academic Editor: Jean-François Berret

Received: 23 February 2021

Accepted: 12 March 2021

Published: 18 March 2021

Publisher's Note: MDPI stays neutral with regard to jurisdictional claims in published maps and institutional affiliations.

Copyright: (c) 2021 by the authors. Licensee MDPI, Basel, Switzerland. This article is an open access article distributed under the terms and conditions of the Creative Commons Attribution (CC BY) license (https:// creativecommons.org/licenses/by/ $4.0 /)$.

\begin{abstract}
Porous scaffolds made of biocompatible and environmental-friendly polymer fibers with diameters in the nano/micro range can find applications in a wide variety of sectors, spanning from the biomedical field to textiles and so on. Their development has received a boost in the last decades thanks to advances in the production methods, such as the electrospinning technique. Conferring antimicrobial properties to these fibrous structures is a primary requirement for many of their applications, but the addition of antimicrobial agents by wet methods can present a series of drawbacks. In this work, strong antibacterial action is successfully provided to electrospun polycaprolactone (PCL) scaffolds by silver (Ag) addition through a simple and flexible way, namely the sputtering deposition of silver onto the PCL fibers. SEM-EDS analyses demonstrate that the polymer fibers get coated by Ag nanoparticles without undergoing any alteration of their morphological integrity upon the deposition process. The influence on wettability is evaluated with polar (water) and non-polar (diiodomethane) liquids, evidencing that this coating method allows preserving the hydrophobic character of the PCL polymer. Excellent antibacterial action (reduction $>99.995 \%$ in $4 \mathrm{~h}$ ) is demonstrated against Escherichia coli. The easy fabrication of these PCL-Ag mats can be applicable to the production of biomedical devices, bioremediation and antifouling systems in filtration, personal protective equipment (PPE), food packaging materials, etc.
\end{abstract}

Keywords: antimicrobial; polycaprolactone (PCL); silver nanoparticles; nanofibers; electrospinning; sputtering; antibacterial; antiviral; biomedical; bioremediation; antifouling

\section{Introduction}

In the last decade, the development of the electrospinning technique has enabled the fabrication of an unprecedented variety of fibrous scaffolds from synthetic and natural materials. Electrospinning is a polymer processing technique of primary interest in scaffold fabrication due to its ability to seamlessly produce fibers with diameters in the range from micrometers down to tens of nanometers, high surface area to volume ratio structure, high porosity and capability to mimic the extracellular matrix (ECM), needful requirements in several applications such as medicine, filtration, textiles, etc. [1-5].

The desirable characteristics of an ideal scaffold are highly dependent on the used polymer and on the given application. Among polymeric materials, Poly( $\varepsilon$-caprolactone) (PCL) is a linear aliphatic polyester with good biocompatibility, ECM mimicking possibility, bioresorbability, biodegradability, thermal stability, mechanical strength, elasticity, 
non-toxicity, slow degradability and low cost, which are desirable characteristics for tissue engineering applications, food packaging, drug delivery systems, wound dressings, filtration and antibacterial constructs [5-8]. However, PCL-derived materials have no antimicrobial properties, unless further functionalized, for example with silver.

Silver $(\mathrm{Ag})$ is one of the most used antimicrobial materials, with biocide action reported against a wide range of bacterial species as well as viruses, so that Ag nanoparticles (NPs) are often added in composite materials to confer them antibacterial properties [9]. Ag-functionalized PCL films are usually produced from a starting solution containing both Ag NPs and PCL mixed together, which is then processed by casting $[10,11]$ or electrospinning [12-14] methods. In spite of the convenience of such procedures, they present some drawbacks. For example, the hydrophilicity of chemically synthesized Ag NPs and the hydrophobicity of PCL can result in composite films with non-uniform NPs dispersion and irregular ion release phenomena for certain applications $[10,11]$. Hence, in situ chemical routes are often employed to synthesize Ag NPs within the same deposition solution [11-13], which, however, can still present many inconveniences, like: addition of a further step in the process, more difficult electrospinning process optimization with the biphasic system (Ag precursor and PCL) with respect to the simpler PCL only, use of toxic and corrosive $\mathrm{Ag}$ precursors, control of $\mathrm{Ag}$ concentration that is conditional on the complete precursor reduction, and use of additional co-solvents to avoid precipitation and favor chemical reaction [11-13]. Additionally, the hydrophilic Ag NPs in the host polymer matrix can shift the PCL hydrophobic character towards a more hydrophilic one $[11,14]$, which could be undesirable under certain circumstances. Finally, starting from a mixed blend of PCL and Ag, only a limited amount of silver is directly exposed on the fiber surfaces and it is not possible to achieve selective functionalization of some desired portions of the membrane only.

As an alternative method, electrospun PCL mats can be post-processed through subsequent $\mathrm{Ag}$ addition by dip coating in silver nitrate aqueous solution, followed by drying and UV photoreduction [15]. Yet, this wet route still presents issues related to wettability mismatches (as observed in [15]), use of unsafe precursors, expected complete reduction of precursors, and impregnation of the whole mat with the Ag solution.

Our study addresses a different strategy, consisting in the addition of Ag nanoparticles onto electrospun bare PCL mats by the sputtering technique, which overcomes many of the drawbacks mentioned above and provides a modular manufacturing route of broader applicability, thus representing a simple and flexible way to functionalize the starting polymer scaffold. Sputtering is an eco-friendly, relatively cheap, widely commercially and industrially employed PVD (physical vapor deposition) method, allowing the deposition of several kinds of materials even on large areas. By properly setting the deposition parameters, sputtering is capable to deposit antibacterial coatings even on delicate materials like soft polymers and bioplastics without significantly damaging them [16]. Sputter deposition of antimicrobial materials, also combining multiple elements together, allows the production of uniform and adherent nanostructured coatings, reducing possible release of particles harmful to human health, and permitting easy and accurate control of the amount of deposited material by tuning the process parameters, like deposition time or power $[17,18]$. Of course, being decoupled from the initial PCL electrospinning process, the sputtering step can be even applied on commercial PCL mats and third-party substrates. Additionally, the physical process of Ag NPs sputter-deposition can confer a slightly hydrophobic character to the treated substrate, as already shown in a previous paper on a different polymer substrate [19], being useful when needing to retain the hydrophobicity of the PCL surface. As a further advantage our method could provide Ag NPs deposited onto the fiber surfaces, promoting the direct exposure of the active antibacterial agent to the surrounding environment. Finally, Ag NPs are deposited in the shallow regions rather than deep within the mat and, by proper masking, they can be placed on selected areas only, thus avoiding deposition/contamination where no functionalization is required. 
So far, only few works reported the functionalization of electrospun PCL-containing scaffolds by the Ag sputtering process [20,21]. However, in those works poly(glycerol sebacate) (PGS) was used as base polymer, while PCL was added to allow the PGS electrospinnability, and their main focus was on the electrical and thermomechanical properties. Only the second report [21] presented some antibacterial results, through the disk diffusion method, on PGS/PCL coated with Ag films with thickness from 50 to $275 \mathrm{~nm}$.

Hence, in the present work, we functionalized the surface of electrospun PCL fibers by sputtering a nanoscale silver coating in the form of Ag nanoparticles, endowing the polymer material with marked antibacterial activity despite a very low Ag content. The results presented hereafter demonstrate the production of PCL-Ag mats through an easy, flexible and effective way, avoiding the disadvantages affecting other methods described above, with possible applications in biomedical devices, food packaging, and sustainability applications like bioremediation and antifouling coatings in filtration. In particular, this class of PCL electrospun sheets have been previously demonstrated to have potential for bioremediation and the production of industrial enzymes and organic acids [22,23]. The first report [22] demonstrated that bare (i.e., uncoated) PCL meshes are highly susceptible to be colonized by pathogenic relevant bacteria, which can be exploited in chronic wound environment to remove biofilms from the wound, thus helping with cleaning and disinfection. In the second study [23], the authors pointed out the satisfactory resistance of these PCL sheets to microbial degradation (compared to polylactic acid) and their ability to support a dense biofilm of pure S. fuliginis (former Flavobacterium sp. ATCC 27551), a very relevant strain in bioremediation of organophosphorus compounds. However, those authors concluded that a functionalization of such PCL sheets would be needed to properly modulate or inhibit microbial activity in many applications, such antifouling surfaces. The coating treatment proposed in this paper also addresses this need.

\section{Materials and Methods}

\subsection{Electrospinning of PCL Scaffolds}

PCL microfibrous sheets with a thickness of $150 \mu \mathrm{m}$ were produced via electrospinning by company Nanofaber srl (Rome, Italy) using a protocol derived by the commercially available grade (NBARE ${ }^{\mathrm{TM}}$ series) to increase the percentage of submicrometer fibers. Process parameters for this batch are reported in Table 1 . The sheets were fabricated using a standard needle-technology electrospinning equipment (Fluidnatek LE100, Bioinicia, Spain) outfitted with a flat collector and a two axes emitter motion to process at room temperature a $12 \% w / v$ solution of PCL polymer dissolved in a mixture solvent of DMF/chloroform 2:8. To prepare the electrospinning solution, pure PCL granules of CAPA ${ }^{\circledR} 6800(80,000$ MW, Perstorp, Sweden) were stirred as long as needed for complete dissolution in said solvent made of dimethylformamide (100\% purity, VWR, Radnor, PA, USA) and chloroform (99.2\% purity, stabilized with $0.6 \%$ ethanol, VWR, Radnor, PA, USA).

Table 1. Process parameters used for the electrospinning of PCL solution $12 \% w / v$.

\begin{tabular}{ccccc}
\hline $\begin{array}{c}\text { Flow Rate } \\
(\mu \mathrm{L} / \mathbf{h})\end{array}$ & $\begin{array}{c}\text { Applied } \\
\text { Voltage }(\mathbf{k V})\end{array}$ & $\begin{array}{c}\text { Needle Inner } \\
\text { Diameter }(\mathbf{m m})\end{array}$ & $\begin{array}{c}\text { Working } \\
\text { Distance (cm) }\end{array}$ & $\begin{array}{c}\text { Deposition } \\
\text { Time (min) }\end{array}$ \\
\hline 4000 & 25 & 1.8 & 17 & 30 \\
\hline
\end{tabular}

Finally, the PCL sheets were mechanically die-cut into disks of $15 \mathrm{~mm}$ diameter to fit in the multiwell plates used for the biological assessment.

\subsection{Sputter-Deposition of Ag Nanoparticles}

The electrospun PCL disks, together with reference silicon substrates, were placed in the vacuum chamber evacuated to a base pressure of $1.5 \times 10^{-4} \mathrm{~Pa}$ for $\mathrm{Ag}$ deposition by rf magnetron sputtering. The deposition was carried out at room temperature in Ar atmosphere at working pressure of $3 \mathrm{~Pa}$. Ag target (purity 99.99\%, diameter $10 \mathrm{~cm}$ ) was 
sputtered by a low power of $50 \mathrm{~W}$ to avoid damaging the PCL fiber bundles with the deposition bombardment. The substrates were rotated by a planetary rotation system at $5 \mathrm{rpm}$, passing under the target plasma for 11 cycles. The nominal thickness of silver deposited on reference $S i$ substrate was $6 \mathrm{~nm}$, which, considering the substrate coverage deduced from SEM inspection, corresponds to Ag load of about $4.4 \mu \mathrm{g} / \mathrm{cm}^{2}$.

\subsection{Scanning Electron Microscopy (SEM) and Energy Dispersive X-ray Spectroscopy (EDS)}

The morphological properties of PCL electrospun meshes were examined with a field emission gun scanning electron microscope Leo 1530 model (ZEISS, Jena, Germany) working at low voltage $(2 \mathrm{kV})$ to avoid charging effects and damage to the dielectric PCL polymer from overheating. Sheet thickness was also measured via SEM on crosssection samples.

Fiber distribution was obtained by measuring the diameter of fibers intersecting a grid sufficiently spaced to accommodate at least 50 intercepting fibers. The SEM image was pre-processed using "ImageJ" software (Rasband, W.S., ImageJ, U.S. National Institutes of Health, Bethesda, MD, USA) to convert images in black \& white.

Energy dispersive X-ray spectroscopy (EDS) measurements were taken using a X-MAX detector (Oxford, UK). PCL sheets samples were examined to map the distribution of Ag on coated samples, however the operating voltage of the SEM was raised to $5 \mathrm{kV}$ to observe Ag peaks in the EDS spectrum.

\subsection{Porosity of PCL Scaffolds}

Gravimetric technique was used for measuring the porosity of electrospun samples. Using a balance (ORMA, BCA120) with precision of $10^{-4} \mathrm{~g}$, knowing the volume of the given sheet sample, the apparent density $\rho *$ of PCL sheets was calculated as:

$$
\rho *=\text { measured mass } / \text { given volume }
$$

The scaffold porosity $\varepsilon$ was obtained using the formula:

$$
\varepsilon=1-\rho * / \rho
$$

upon assuming a value of $\rho=1.145 \mathrm{~g} / \mathrm{cm}^{3}$ for PCL. Porosity measurements were replicated at least three times for each type of samples, with reported $\varepsilon$ being the average.

\subsection{Contact Angle Measurements}

Wetting tests were performed on a contact angle goniometer (OCA 20, Dataphysics, Filderstadt, Germany) operating at room temperature. The contact angle was measured by the sessile drop method using $1 \mu \mathrm{L}$ droplet volume and deposition rate of $1 \mu \mathrm{L} / \mathrm{s}$. Milli-Q water and diiodomethane were used as the polar and the apolar substances, respectively. The images were captured after 10,60 and $120 \mathrm{~s}$ and data were collected with SCA 202 software (version 3.4.3 build 76). For statistical accountability, the average of ten contact angle measurements for each sample was calculated.

\subsection{Antimicrobial Tests}

Escherichia coli (E. coli) stock cultures kept at $-80{ }^{\circ} \mathrm{C}$ in $10 \%$ (wt./vol.) glycerol were inoculated into $5 \mathrm{~mL}$ of Luria-Bertani (LB) broth and incubated at $37{ }^{\circ} \mathrm{C} \mathrm{O} / \mathrm{N}$ before their use in experiments. E. coli was pre-inoculated aerobically for $16 \mathrm{~h}$ at $37^{\circ} \mathrm{C}$ in LB medium, with constant shaking at $250 \mathrm{rpm}$. The day of the test the bacteria were diluted and grown until OD600 was 0.025 , about $5.5 \times 10^{6}$ colony forming units $/ \mathrm{mL}(\mathrm{CFU} / \mathrm{mL}$ ). Then the bacteria were placed in multiwell plate with 24-wells in the presence of uncoated PCL samples (blank disks) or Ag-coated samples and incubated at $37^{\circ} \mathrm{C}$ under constant agitation at $50 \mathrm{rpm}$. A control with E. coli alone was also inserted. At following times $t=0$, 2 and $4 \mathrm{~h}, 10 \mu \mathrm{L}$ of each sample were diluted in PBS (serial dilution from $10^{-1}$ to $10^{-4}$ ) and $10 \mu \mathrm{L}$ of each dilution were distributed on LB agar dishes $\left(15 \mathrm{~g} \mathrm{~L}^{-1}\right.$ agar) and incubated 
for $18 \mathrm{~h}$ at $37^{\circ} \mathrm{C}$. Each plating was performed in triplicates. Subsequently, the number of $\mathrm{CFU} / \mathrm{mL}$ was quantified for each sample. The experiment was repeated twice.

\section{Results and Discussion}

Figure 1 shows the picture of some representative samples of electrospun blank PCL disks as obtained from the electrospinning process, and PCL-Ag disks from the subsequent sputter-deposition of Ag on the electrospun PCL mat.

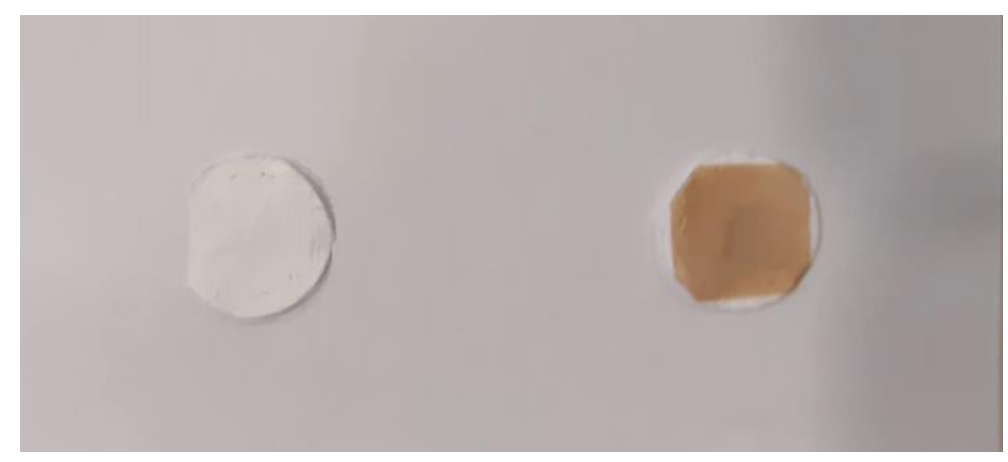

Figure 1. Picture of representative samples of electrospun blank PCL (left) and Ag-coated PCL (right).

\subsection{SEM-EDS Analyses}

The samples microstructure was characterized by SEM. The membrane, imaged at low magnification, appears macroscopically dense, as a thick maze of intertwined randomly oriented fibers. The diameter of the fibers is broadly distributed from $100 \mathrm{~nm}$ or less to some microns. The fiber diameter distribution as determined by fiber counting on SEM micrographs was $1.83 \pm 0.54 \mu \mathrm{m}$ and the average porosity $80.9 \pm 1.8 \%$. The morphology of the PCL sheets before and after the Ag treatment is displayed in the micrographs of Figure 2. It appears essentially identical apart from the higher contrast in the treated PCL, reflecting a higher SEM signal from the Ag metallization. Remarkably, this indicates that the proposed coating process is non-destructive and sufficiently gentle to be performed on a temperature sensitive polymer such as PCL (melting temperature about $50-60{ }^{\circ} \mathrm{C}$ ).

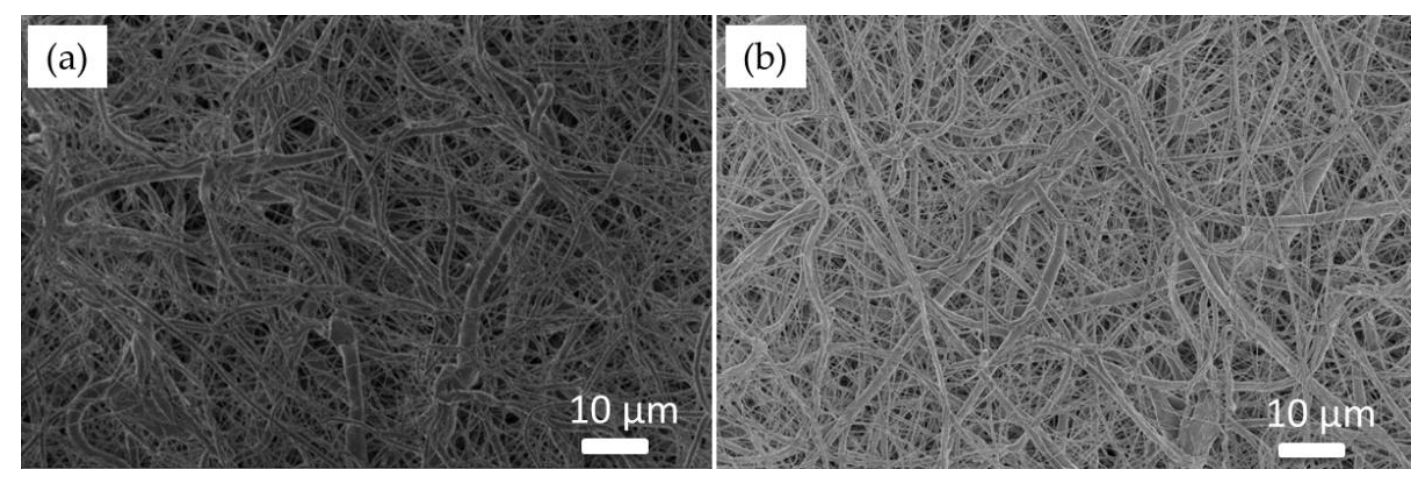

Figure 2. SEM micrographs at low voltage showing an overview at low magnification of (a) the bare sample vs. (b) Ag-coated one.

The close-up micrographs in Figure 3 further demonstrate that the coating process does not alter morphology and texture, while revealing the Ag particles decorating the PCL fibers. From the images of the coated fibers it was possible to identify Ag particles with lateral dimensions ranging from a few nanometers to some ten of nanometers, together with some bigger clusters up to around $80 \mathrm{~nm}$. From particle counting on the SEM micrographs, the average nanoparticle diameter was determined to be $(22 \pm 9) \mathrm{nm}$, which, however, does not take into account particles smaller than about $9 \mathrm{~nm}$, as they cannot be correctly measured due to image resolution at the given magnifications. 


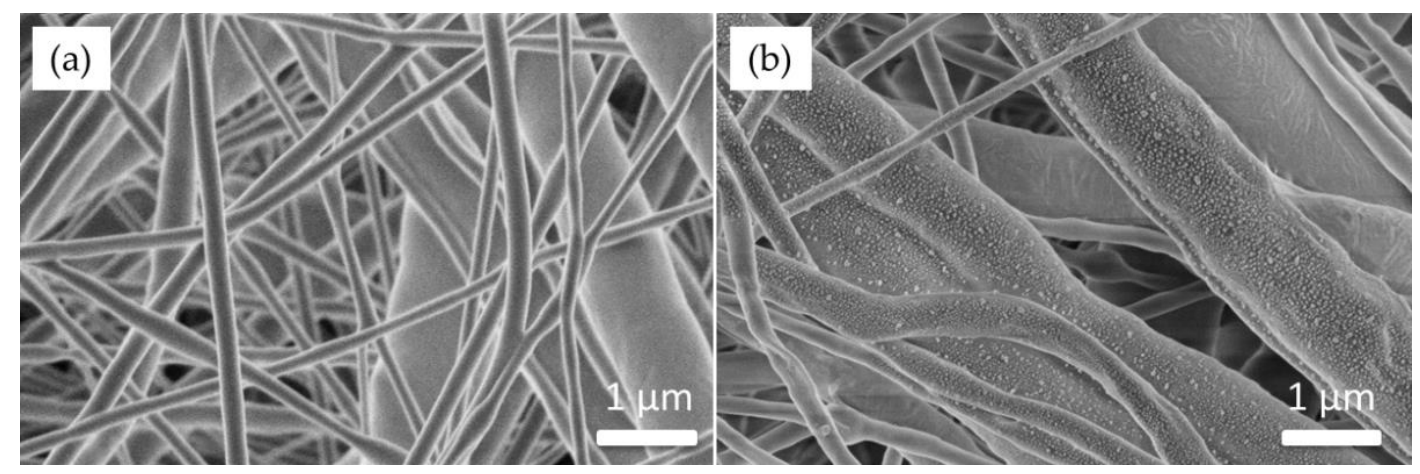

Figure 3. Close-up SEM micrographs at low voltage showing (a) the fibers in the bare sample vs. (b) fibers of the Ag-coated one, where silver decoration is clearly visible.

The absence of any evident damage (modifications in fibers shape or dimension, fiber fusion or collapse, occlusion of pores, etc.) in our conditions is also noteworthy when looking to analogous experimental findings for Ag deposition onto electrospun polymer fibers. In a recent paper [24], a different PVD process was employed to coat various polymer fibers, finding no damages on some polymers while important alterations and degradation on another. Such effects may depend on the polymeric substrate properties, the amount of silver deposited, as well as the characteristics of the deposition process (deposition technique and process parameters employed) that influence the energy of the depositing species and the consequent impact on the coated fibers. In our case, the suitable combination of deposition technique, process parameters and amount of deposited silver allowed the functionalization of the soft PCL substrate without inducing significant damages. At the same time, the kinetic energy of the sputtered Ag particles impinging on the soft polymer fibers is expected to promote a good adhesion of the silver nanocoating, preventing their premature detachment and release of large amount of particles that could result harmful for living organisms. Although these aspects need ad hoc analyses to get an exact evaluation, which could be object of future works, a preliminary rough hint on coating stability has been here deduced from the absence of any evident visual damage on the coated samples during their manipulation and after intentional manual bending and attempts to scratch them.

Since Ag was deposited as a nanoparticle layer around the fiber surfaces, it is not expected to significantly and directly modify the through-thickness capillary properties of the PCL membrane, which are important in filtering applications. While not verified by experimental tests in our study, this expectation is supported by models for disordered filtering media found in the literature, such as the work based on fractal theory reported in [25], linking the overall capillary flow to the through-thickness properties and, thus, suggesting that the nanoscale surface modification in our PCL-Ag scaffolds should not be detrimental in that regard.

The presence of silver on the surface of the PCL fibers was also confirmed by EDS mapping, as visible in Figure 4. Ag signal coming from the fiber bundle (Figure 4a) was detected in the EDS spectra (Figure 4b), and it was found to be distributed along the surface of the single fibers, as shown in Figure 4c-e.

SEM inspection of Ag deposited on reference silicon substrates, coated together with the PCL disks during the same process, was used to estimate the Ag content and demonstrate the formation of an incoherent nanoscale coating with the chosen sputtering parameters, that is both functional and "delicate" enough for polymer applications from a technological perspective. Low magnification micrograph in Figure 5a shows a uniform distribution of Ag particles over the substrate surface. Looking to the higher magnification (Figure $5 b$ ), the fine morphology appears constituted by islands formed as a results of silver atoms and ions coalescing on the silicon substrate during the deposition process, as discussed in previous work [19]. 

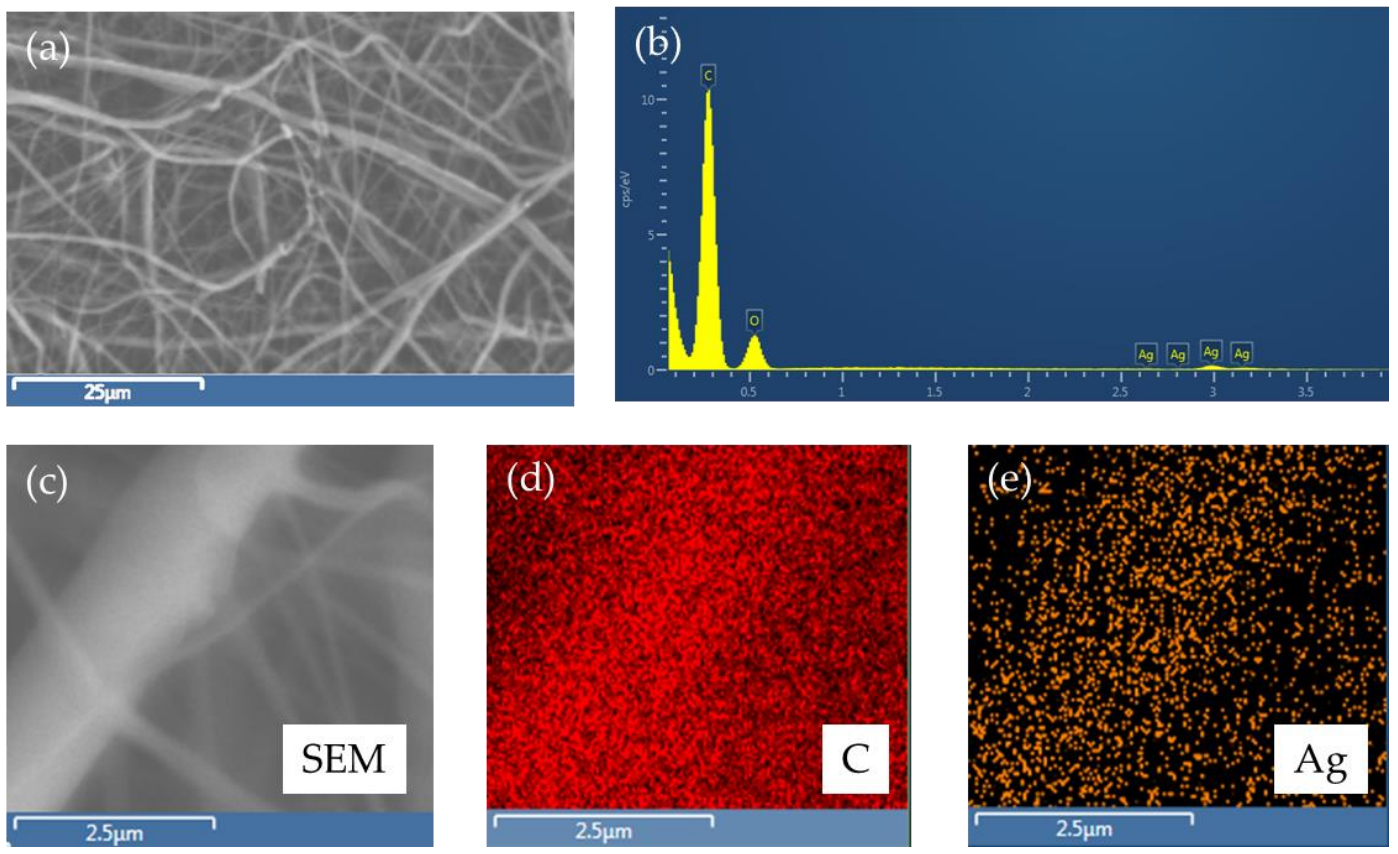

Figure 4. EDS mapping confirming presence of Ag on the Ag-coated PCL samples obtained at $5 \mathrm{kV}$ operating voltage. $(\mathbf{a}, \mathbf{b})$ SEM and related EDS spectrum taken on a fiber bundle region; (c-e) SEM and related EDS mapping taken on a single fiber.
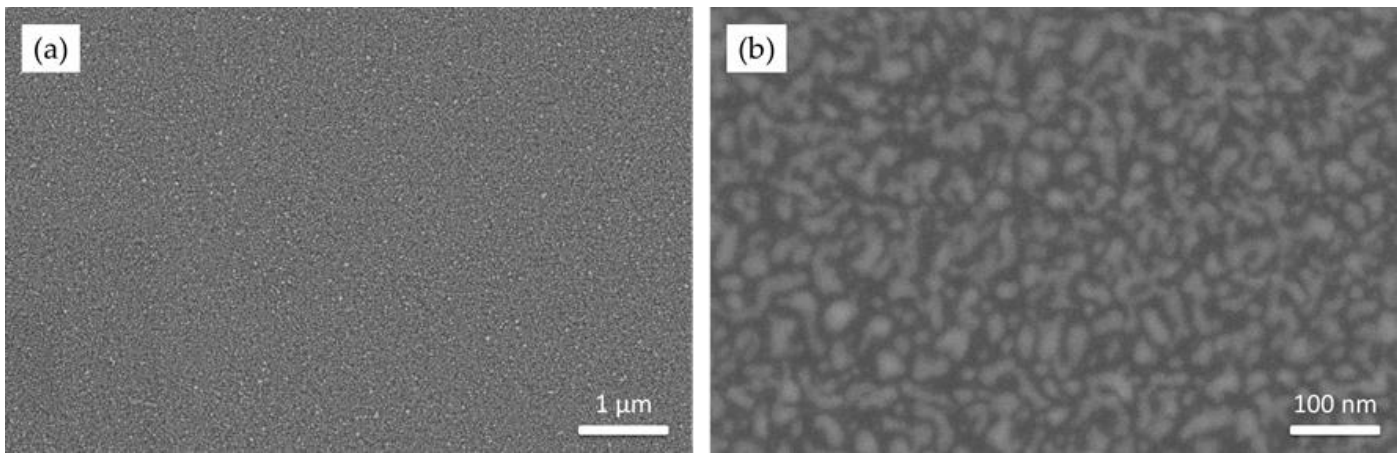

Figure 5. SEM images at (a) low and (b) high magnification of silver coating deposited on reference silicon substrate.

Measuring the substrate coverage $\alpha$ from high magnification SEM images, it was possible to estimate the Ag load per unit surface (concentration $C$ in $\mu \mathrm{g} / \mathrm{cm}^{2}$ ) deposited on the samples, as:

$$
C=\rho \times \alpha \times t,
$$

where $\rho$ is the silver density and $t$ is the Ag coating thickness. By assuming the theoretical Ag density $\rho=10.49 \mathrm{~g} / \mathrm{cm}^{3}$, the coating thickness $t=6 \mathrm{~nm}$ as measured by atomic force microscopy [19], and $\alpha=0.7$ (being the effective coated surface around $70 \%$ as per SEM inspection), the specific density of the deposited Ag coating in our samples was about $4.4 \mu \mathrm{g} / \mathrm{cm}^{2}$, corresponding to a remarkably low Ag load.

\subsection{Wettability Analyses}

The wettability of the blank and Ag-functionalized electrospun samples (PCL and PCL-Ag, respectively) was determined by contact angle measurements with water and diiodomethane. The photographs of representative measurements are shown in Figure 6, while the water contact angles (WCA) are presented in Table 2. 

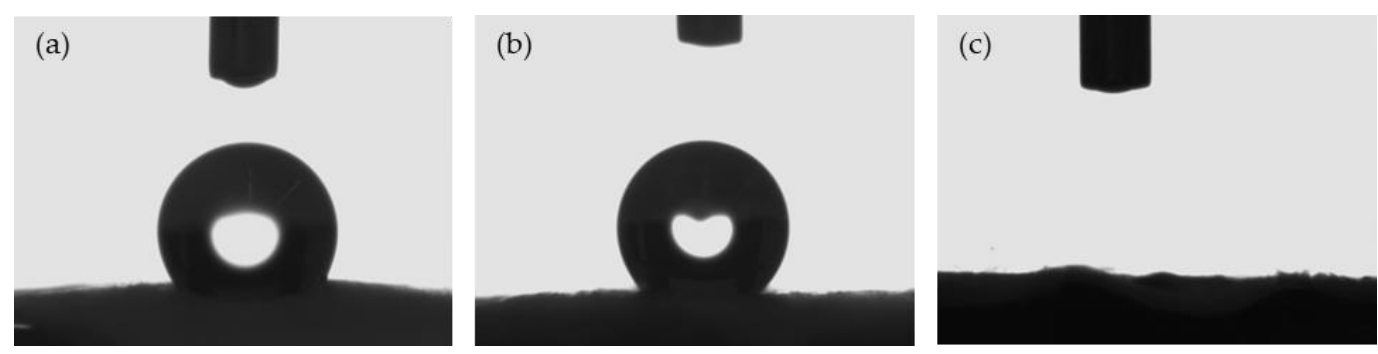

Figure 6. Photographs of water droplet on PCL surface before (a) and after Ag deposition (b). Photograph of diiodomethane droplet on blank PCL surface (c), as representative of both PCL and PCL-Ag surfaces.

Table 2. Water contact angles (mean \pm standard deviation) of PCL and PCL-Ag samples.

\begin{tabular}{cc}
\hline Sample & WCA $\left(^{\circ}\right)$ \\
\hline PCL & $128.7 \pm 4.7$ \\
PCL-Ag & $134.3 \pm 2.6$ \\
\hline
\end{tabular}

The pure PCL fibrous mats showed a water contact angle around $129^{\circ}$, indicating the hydrophobic nature of the studied PCL scaffolds, as expected due to the presence of $\mathrm{CH}_{2}$ groups in the backbone of PCL chains, that is the main reason for its hydrophobic character [26]. After the Ag NPs deposition on the surface of the PCL fibers, a slight increase in the contact angle was observed, resulting in a measured WCA value around $134^{\circ}$. The WCA was measured after 10, 60 and $120 \mathrm{~s}$ and no variations of the drop shape were observed. The apolar behavior of PCL and PCL-Ag was confirmed by the full permeability displayed when diiodomethane was used to perform the test. In this case, the fluid was fully absorbed by both the uncoated and Ag-coated PCL fibers (Figure 6c).

It is worth noting that the slight increase of WCA after Ag addition by means of physical deposition techniques is consistent with results previously reported on polylactide (PLA) substrates [19]. This is particularly interesting when compared with Ag modification of PCL scaffolds by chemical-based routes, where the presence of silver turned the PCL wettability from hydrophobic to hydrophilic [11,14]. Thus, physical deposition of Ag NPs onto PCL fibers by the sputtering technique represents a successful method to retain the hydrophobic character in the final composites.

\subsection{Antimicrobial Tests}

The antimicrobial activity of the uncoated and Ag-coated PCL samples was tested for Gram-negative bacteria E. Coli. The samples were incubated with the microbial population, and the survival rate was determined at 2 and $4 \mathrm{~h}$, using the count plate method and calculation of CFUs to determine the cytocitic action of the samples. Photographs of the Petri dishes obtained from control culture (E. coli in the absence of any mat) and from cultures with blank PCL and PCL-Ag mats at different serial dilutions and different sampled times are reported in Figure 7. Data obtained from the CFU calculations are listed in Table 3 and plotted in the top right panel of Figure 7. 
$\mathrm{Oh}$

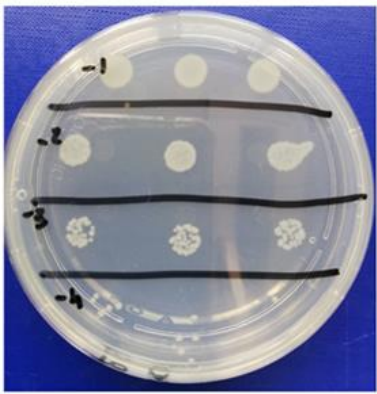

Control
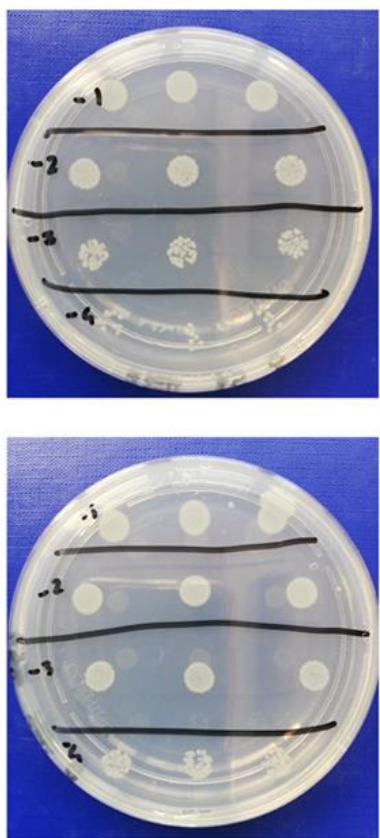

Control

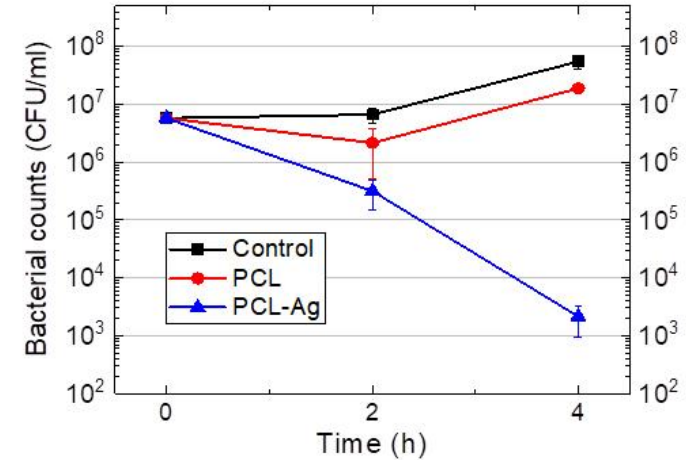

PCL
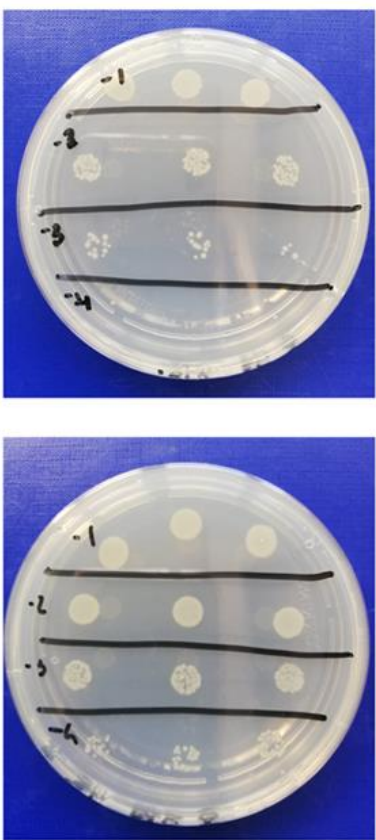

PCL
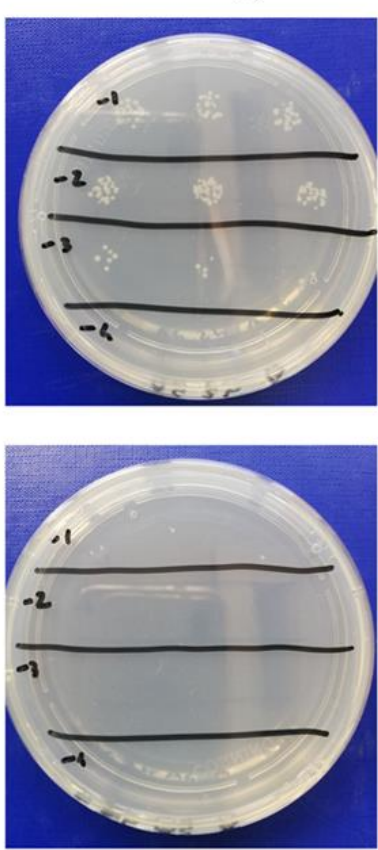

PCL-Ag

Figure 7. Photographs of Petri dishes obtained from E. coli culture without any mat (Control) and cultures with blank PCL and PCL-Ag mats at different serial dilutions from $10^{-1}$ to $10^{-4}$ (writing on the covers) and different sampled times ( 0,2 and $4 \mathrm{~h})$. Top right panel: graph of data obtained from the CFU calculations.

Table 3. Bacterial population (CFU/mL) measured for control, uncoated sample (PCL) and coated sample (PCL-Ag) at different times. Data are reported as mean \pm standard deviations from two independent experiments, each in triplicate.

\begin{tabular}{cccc}
\hline & \multicolumn{3}{c}{ Bacterial Population (CFU/mL) } \\
\cline { 2 - 4 } Sample & $\mathbf{0 ~ h}$ & $\mathbf{2} \mathbf{h}$ & $\mathbf{4} \mathbf{h}$ \\
\hline Control & $(5.8 \pm 1.1) \times 10^{6}$ & $(6.6 \pm 1.9) \times 10^{6}$ & $(5.5 \pm 1.4) \times 10^{7}$ \\
PCL & - & $(2.1 \pm 1.6) \times 10^{6}$ & $(1.9 \pm 0.1) \times 10^{7}$ \\
PCL-Ag & - & $(3.1 \pm 1.6) \times 10^{5}$ & $(2.1 \pm 1.2) \times 10^{3}$ \\
\hline
\end{tabular}

As expected, E. Coli culture in the absence of any mat, used as a control, kept growing up to $4 \mathrm{~h}$, passing from the initial concentration of $5.8 \times 10^{6}$ to $5.5 \times 10^{7} \mathrm{CFU} / \mathrm{mL}$ at $4 \mathrm{~h}$. It was observed that the blank PCL sample induced a slight reduction in bacterial growth, leading to a 3-fold decrease of bacterial population with respect to the control. This weak antimicrobial action remained constant in the considered time range, indicating that the asgrown bare PCL mat represented a slightly unfavorable environment for bacterial growth, 
probably due to hindering in bacterial cells reproduction and to mechanical detrimental effects (e.g., cell deformation and wall abrasion caused by the interactions between the bacterial cells and the material surface). Interestingly, PCL-Ag sample showed good antimicrobial action already in the first $2 \mathrm{~h}$ of treatment, by reducing $E$. coli cellular density of almost $1 \log$ (about 7 times) compared to the blank PCL sample and more than 1 Log (about 20 times) compared to the control population. After $4 \mathrm{~h}$ the inhibitory effect of PCL-Ag on bacterial grown was excellent, leading to a decrease of almost 4 Log compared to blank PCL and almost 4.5 Log compared to the control. Such strong antibacterial activity was reached in few hours and by using a very lower amount of silver in comparison to previous findings [21] (nominal thickness of $6 \mathrm{~nm}$ vs. $50-275 \mathrm{~nm}$ ). This can be a consequence of the Ag nanoparticle deposition achieved on the PCL fibers in our samples, instead of a uniform film coverage of the fibers, since nanostructuration leads to increase the effective surface of antibacterial agent exposed to the environment and the consequent antimicrobial efficacy [9]. Moreover, it is worth noting that the antibacterial effect in our PCL-Ag scaffolds resulted faster and higher with respect to composites produced starting from a mixed blend [11,13,27], despite the Ag content ratio in our samples (approximately $0.1 \mathrm{wt} . \%$ with respect to PCL) is comparable or even well below the one used in previous works, as expected due to the presence of the Ag nanoparticles directly on the surfaces of the PCL fibers in our method rather than incorporated within the fibers. More importantly, it must be underlined that, since we are dealing with Ag coating the fiber surfaces, the most correct parameter to be taken into account is essentially the Ag surface load in our PCL-Ag mats, that is as low as $\sim 4 \mu \mathrm{g} / \mathrm{cm}^{2}$, being able to confer the very effective antimicrobial action here demonstrated.

\section{Conclusions}

In this work we proposed the preparation of antibacterial mats through deposition of Ag NPs by sputtering silver onto the surface of electrospun PCL scaffolds. SEM analyses demonstrated that the Ag deposition process did not alter the overall morphology and texture of the original PCL mats characterized by randomly oriented fibers. The presence of Ag nanoparticles, with average size around $20 \mathrm{~nm}$, decorating the surface of the PCL fibers was evident in SEM images and confirmed by EDS mapping. The wettability tests revealed that the hydrophobic character of the polymer was preserved in the final PCL-Ag composite. The Ag-coated PCL mats exhibited high antibacterial effect against gramnegative E. coli bacterial strain. This strong antimicrobial action obtained despite the low $\mathrm{Ag}$ content is ascribable to the increased effective surface of the antibacterial agent, thanks to the nanostructuration of silver in form of nanoparticles directly deposited on the surfaces of the PCL fibers.

On the basis of the PCL polymer properties, of the peculiar characteristics of the electrospun fiber scaffolds and of the antimicrobial activity provided by means of this straightforward method, the properties of the PCL-Ag systems developed and the proposed method can find promising exploitation in different applications, such as biomedical devices, medical PPE, food packaging, bioremediation and antifouling coatings in filtration.

The results here presented demonstrate that PCL can be successfully treated through an easy, flexible and effective method for the production of Ag-functionalized PCL mats endowed with interesting antimicrobial properties, as advocated in prior studies [22,23]. In order to confirm the practical usage of the materials here developed for the proposed applications, further analyses are expected to evaluate other important aspects, such as particle release phenomena, cytocompatibility with human cells, and coating stability.

Author Contributions: Conceptualization, D.V. and A.R.; methodology, D.V., L.T., R.V., G.G. and A.R.; investigation, D.V., L.T., R.V., G.G. and A.R.; writing-original draft preparation, D.V., L.T., R.V. and A.R.; writing-review and editing, D.V., L.T., R.V. and A.R. All authors have read and agreed to the published version of the manuscript.

Funding: This research received no external funding. 
Institutional Review Board Statement: Not applicable.

Informed Consent Statement: Not applicable.

Data Availability Statement: Data available on request from the corresponding authors.

Conflicts of Interest: The authors declare the following competing financial interest(s): The authors A.R. and G.G. are affiliated with the company Nanofaber srl. This study is not a funded or sponsored research work.

\section{References}

1. Shin, Y.M.; Hohman, M.M.; Brenner, M.P.; Rutledge, G.C. Electrospinning: A whipping fluid jet generates submicron polymer fibers. Appl. Phys. Lett. 2001, 78, 1149-1151. [CrossRef]

2. Teo, W.E.; Ramakrishna, S. A review on electrospinning design and nanofibre assemblies. Nanotechnology 2006, 17, R89-R106. [CrossRef]

3. Mirjalili, M.; Zohoori, S. Review for application of electrospinning and electrospun nanofibers technology in textile industry. J. Nanostruct. Chem. 2016, 6, 207-213. [CrossRef]

4. Nitti, P.; Gallo, N.; Palazzo, B.; Sannino, A.; Polini, A.; Verri, T.; Barca, A.; Gervaso, F. Effect of L-Arginine treatment on the in vitro stability of electrospun aligned chitosan nanofiber mats. Polym. Test. 2020, 91, 106758. [CrossRef]

5. Haider, A.; Haider, S.; Kang, I.-K. A comprehensive review summarizing the effect of electrospinning parameters and potential applications of nanofibers in biomedical and biotechnology. Arab. J. Chem. 2018, 11, 1165-1188. [CrossRef]

6. Woodruff, M.A.; Hutmacher, D.W. The return of a forgotten polymer-Polycaprolactone in the 21st century. Prog. Polym. Sci. 2010, 35, 1217-1256. [CrossRef]

7. Mohamed, R.M.; Yusoh, K. A Review on the Recent Research of Polycaprolactone (PCL). Adv. Mater. Res. 2015, 1134, 249-255. [CrossRef]

8. Guarino, V.; Gentile, G.; Sorrentino, L.; Ambrosio, L. Polycaprolactone: Synthesis, Properties, and Applications. In Encyclopedia of Polymer Science and Technology; John Wiley and Sons, Inc.: Hoboken, NJ, USA, 2017; pp. 1-36.

9. Khezerlou, A.; Alizadeh-Sani, M.; Azizi-Lalabadi, M.; Ehsani, A. Nanoparticles and their antimicrobial properties against pathogens including bacteria, fungi, parasites and viruses. Microb. Pathog. 2018, 123, 505-526. [CrossRef]

10. Koutsoumpis, S.; Poulakis, A.; Klonos, P.; Kripotou, S.; Tsanaktsis, V.; Bikiaris, D.N.; Kyritsis, A.; Pissis, P. Structure, thermal transitions and polymer dynamics in nanocomposites based on poly( $\varepsilon$-caprolactone) and nano-inclusions of $1-3 \mathrm{D}$ geometry. Thermochim. Acta 2018, 666, 229. [CrossRef]

11. Tran, P.A.; Hocking, D.M.; O'Connor, A.J. In situ formation of antimicrobial silver nanoparticles and the impregnation of hydrophobic polycaprolactone matrix for antimicrobial medical device applications. Mater. Sci. Eng. C 2015, 47, 63. [CrossRef]

12. Sumitha, M.S.; Shalumon, K.T.; Sreeja, V.N.; Jayakumar, R.; Nair, S.V.; Menon, D. Biocompatible and antibacterial nanofibrous poly(E-caprolactone)- nanosilver composite scaffolds for tissue engineering applications. J. Macromol. Sci. Part A Pure Appl. Chem. 2012, 49, 131. [CrossRef]

13. Pazos-Ortiz, E.; Roque-Ruiz, J.H.; Hinojos-Márquez, E.A.; López-Esparza, J.; Donohué-Cornejo, A.; Cuevas-González, J.C.; Espinosa-Cristóbal, L.F.; Reyes-López, S.Y. Dose-Dependent Antimicrobial Activity of Silver Nanoparticles on Polycaprolactone Fibers against Gram-Positive and Gram-Negative Bacteria. J. Nanomater. 2017, 2017, 4752314. [CrossRef]

14. Thomas, R.; Soumya, K.R.; Mathew, J.; Radhakrishnan, E.K. Electrospun Polycaprolactone Membrane Incorporated with Biosynthesized Silver Nanoparticles as Effective Wound Dressing Material. Appl. Biochem. Biotechnol. 2015, 176, 2213. [CrossRef]

15. Lim, M.M.; Sultana, N. In vitro cytotoxicity and antibacterial activity of silver-coated electrospun polycaprolactone/gelatine nanofibrous scaffolds. 3 Biotech 2016, 6, 211. [CrossRef]

16. Valerini, D.; Tammaro, L.; Villani, F.; Rizzo, A.; Caputo, I.; Paolella, G.; Vigliotta, G. Antibacterial Al-doped ZnO coatings on PLA films. J. Mater. Sci. 2020, 55, 4830-4847. [CrossRef]

17. Valerini, D.; Tammaro, L.; Di Benedetto, F.; Vigliotta, G.; Capodieci, L.; Terzi, R.; Rizzo, A. Aluminum-doped zinc oxide coatings on polylactic acid films for antimicrobial food packaging. Thin Solid Films 2018, 645, 187-192. [CrossRef]

18. Benetti, G.; Cavaliere, E.; Banfi, F.; Gavioli, L. Antimicrobial nanostructured coatings: A gas phase deposition and magnetron sputtering perspective. Materials 2020, 13, 784. [CrossRef]

19. Valerini, D.; Tammaro, L.; Vigliotta, G.; Picariello, E.; Banfi, F.; Cavaliere, E.; Ciambriello, L.; Gavioli, L. Ag functionalization of Al-doped ZnO nanostructured coatings on PLA substrate for antibacterial applications. Coatings 2020, 10, 1238. [CrossRef]

20. Memic, A.; Aldhahri, M.; Tamayol, A.; Mostafalu, P.; Abdel-Wahab, M.S.; Samandari, M.; Moghaddam, K.M.; Annabi, N.; Bencherif, S.A.; Khademhosseini, A. Nanofibrous silver-coated polymeric scaffolds with tunable electrical properties. Nanomaterials 2017, 7, 63. [CrossRef]

21. Kalakonda, P.; Aldhahri, M.A.; Abdel-Wahab, M.S.; Tamayol, A.; Moghaddam, K.M.; Ben Rached, F.; Pain, A.; Khademhosseini, A.; Memic, A.; Chaieb, S. Microfibrous silver-coated polymeric scaffolds with tunable mechanical properties. RSC Adv. 2017, 7,34331. [CrossRef] 
22. Rumbo, C.; Tamayo-Ramos, J.A.; Caso, M.F.; Rinaldi, A.; Romero-Santacreu, L.; Quesada, R.; Cuesta-López, S. Colonization of Electrospun Polycaprolactone Fibers by Relevant Pathogenic Bacterial Strains. ACS Appl. Mater. Interfaces 2018, 10, 11467-11473. [CrossRef] [PubMed]

23. Tamayo-Ramos, J.A.; Rumbo, C.; Caso, M.F.; Rinaldi, A.; Garroni, S.; Notargiacomo, A.; Romero-Santacreu, L.; Cuesta-López, S. Analysis of Polycaprolactone Microfibers as Biofilm Carriers for Biotechnologically Relevant Bacteria. ACS Appl. Mater. Interfaces 2018, 10, 32773-32781. [CrossRef]

24. Pagnotta, G.; Graziani, G.; Baldini, N.; Maso, A.; Focarete, M.L.; Berni, M.; Biscarini, F.; Bianchi, M.; Gualandi, C. Nanodecoration of electrospun polymeric fibers with nanostructured silver coatings by ionized jet deposition for antibacterial tissues. Mater. Sci. Eng. C 2020, 113, 110998. [CrossRef]

25. Xiao, B.; Huang, Q.; Chen, H.; Chen, X.; Long, G. A fractal model for capillary flow through a single tortuous capillary with roughened surfaces in fibrous porous media. Fractals 2021, 29, 2150017. [CrossRef]

26. Zhang, J.; Qiu, Z. Morphology, crystallization behavior, and dynamic mechanical properties of biodegradable poly( $\varepsilon$ caprolactone)/thermally reduced graphene nanocomposites. Ind. Eng. Chem. Res. 2011, 50, 13885-13891. [CrossRef]

27. Gao, Y.; Hassanbhai, A.M.; Lim, J.; Wang, L.; Xu, C. Fabrication of a silver octahedral nanoparticle-containing polycaprolactone nanocomposite for antibacterial bone scaffolds. RSC Adv. 2017, 7, 10051. [CrossRef] 